\title{
Appraising the Diagnostic and Statistical Manual against Recovery philosophies in elderly dementia
}

\author{
Robyn Aldrich
}

Robyn Aldrich is employed at the Auckland District Health Board as a social work intern currently on placement with Mental Health Services for Older Persons. Robyn is undertaking a Postgraduate Certificate in Health (Allied Mental Health) at Victoria University.

\begin{abstract}
This article looks at the history of the Diagnostic Statistical Manual (DSM) classification system, its role and limitations, while making comparisons and contrasts to Recovery philosophies in relation to elderly dementia from a social work perspective. It was found that the clinician, caregiver and patient can all play a role in Recovery, and while not comparable the DSM complements psychosocial models of Recovery. It was also found that with the diversification and expansion of an increasing ageing population, gerontological social workers will need to modify and adapt practice skills and interventions as changes in evidence-based practices of Recovery and the DSM are disseminated at a faster rate.
\end{abstract}

\section{Introduction}

The New Zealand ageing population is increasing. Statistics New Zealand (2006) state that by 2051 there will be over 1.14 million people aged 65 and over in New Zealand. Of this population, 50,000 will have some form of dementia (Perkins, 2006), and for every person with dementia there will be two people affected by that person's dementia. Dementia is classified as a biological degenerative brain disorder that affects memory, reasoning and judgement. It can also lead to a loss of language, motor activity and a failure to recognise things, which consequently impedes everyday activities and social relationships (Corcoran \& Walsh, 2006). To assist in identifying such disorders, medicine has traditionally used categorisations as a reference tool for physicians. The Diagnostic and Statistical Manual of Mental Disorders (American Psychiatric Association, 2005) (DSM) is one such classification system. This essay will look at the history of the DSM classification system, its role and limitations, while making comparisons with and contrasts to Recovery philosophies with regard to elderly dementia.

\section{Early classification systems}

The initial incentive for a clinical classification system which started in the 1900s, was to collect statistics from mental hospitals across America, and to provide nomenclatures and descriptions of psychiatric and neurological disorders to provide some uniformity. One psychiatric disorder noted at that time was 'Presenile Dementia' discovered by Ger- 
man neuropathologist Alois Alzheimer. Presenile Dementia was later to be established by Alzheimer's colleague Emil Kraepelin as 'Alzheimer's disease' (Neurological Foundation of New Zealand, 2002). The classification system was refined in 1927, 1933 and 1942 respectively, to add disorders, and incorporate new developments in medical research. A paradigm up until the 1940s (Collier, 2008) was that dementia was an expected medical condition of elderly due to hardening of the arteries. As a result dementia was established as untreatable, therefore there was little interest in performing research.

Following World War II the Navy, Army and Veterans Administration revised their own classification system. This was to accommodate presentations of mental disorders by military personnel that did not fit with other nomenclatures. One such example was Post Traumatic Stress Disorder (Collier, 2008). The World Health Organisation also published a classification system, 'International Classification of Diseases' (ICD), as a record of English health statistics. The ICD incorporated psychiatric illness into its sixth edition, however it has been criticised over the years for not being specific enough.

In 1952 a variant of the ICD 6 inclusive of the initial clinical classification system was created and published as DSM-I (American Psychiatric Association, 2005). Each diagnosis had a description followed by symptoms, but did not provide clinicians with treatment approaches. Combining biological with psychological research DSM-I had 106 diagnoses listed (Corcoran \& Walsh, 2006). One of the progressive changes in diagnoses as a result of research was that 'Dementia Praecox' was found to be quite distinct from other forms of dementia. As a result the original term Dementia Praecox was renamed Schizophrenia. This left the only diagnosis applicable to elderly in DSM-I as Senile Dementia (Collier, 2008). By 1968 when DSM-II was published, research had eventually recognised Senile Dementia as a brain disorder, as opposed to an inevitable process of ageing.

\section{DSM reflecting value judgments}

There has been ongoing debate over the years as to whether diagnoses and classifications systems reflect value judgments, for instance 'ageist attitudes'. Glasgow (2005) maintains that elderly are often perceived as, and expected to become, senile, frail, disabled, likely to live in institutions, unable to learn new skills, to be socially withdrawn and dependent. Thompson (2001) supports this, adding that ageism can also reinforce fear and denigration of the ageing process thereby establishing assumptions regarding the ability of older people. This is happening in England presently, where elderly have been excluded from mental health policies (Collier, 2008). Collier considers ageism in the identification of mental disorders arguing that the word 'senile' has negative connotations for elderly and should not be used to describe someone with diminished cognitive abilities. The diagnostic term 'Senile Dementia' has become socially acceptable interchangeably with other terms such as 'old', 'demented' and 'senile' to mean one and the same thing. Thus the development of classification systems can influence people's attitudes within society. Values and responses such as this do not support Recovery (Mental Health Commission, 2001). Therefore social workers in particular (Kirst-Ashman \& Hull, 2006) must work towards eliminating or changing preconceived notions, stereotypes and paternalistic attitudes concerning elderly.

DSM-III published in 1980 introduced a contrast to the ICD in that it provided more comprehensive diagnoses, and was modified for use in the United States. This publication 
(American Psychiatric Association, 2005) provided a multi-axial system which included a psychosocial inventory of problems, subtypes and specifiers, also a more neutral view of aetiology. Obtainable for the first time internationally, the DSM-III was translated into many languages. Although claimed to be less biased (Dziegielewski, 2002) DSM-III revealed many inconsistencies and controversies, such as persistent rape being a disorder as opposed to a criminal act (Corcoran \& Walsh, 2006). Also it was argued that there was not much in the way of evidence-based practice in its diagnostic descriptions. Consequently the American Psychiatric Association (APA) was challenged to test the DSM's reliability with independent researchers that would provide a more critical evaluation. This lead to DSM-III-R in 1987 which was based on field trials leading to 100 categories being affected in some way (American Psychiatric Association, 2005). Despite claims of innovation with each DSM revision, it wasn't until 1987 that psychiatric classifications recognised mental illness in older adults (Collier, 2008). Chiu (2005) however claims the diagnosis of dementia was more specific in this revision than the subsequent DSM-IV.

\section{DSM criticisms, reliability and validity}

With 340 mental disorders within 16 classifications, the DSM-IV published in 1994 incorporated information based on considerable experimental research and clinical experience. DSM-IV attempted to improve on criticisms of previous publications, to include cultural information, and align itself with the ICD 10th edition (Dziegielewski, 2002). However, Kutchins and Kirk (1996) proposed that the DSM-IV still failed to provide convincing evidence of reliability or validity of the diagnostic criteria. Chiu (2005) and Perkins (2006) agree, noting problems with the diagnostic criteria for dementia. The DSM-IV diagnosis of dementia is made on the basis that there must be memory loss, however, sometimes memory loss is not the first symptom presenting, especially with frontotemporal dementia which affects language and emotions. Furthermore, Perkins states that with dementia there are often problems with personality changes, behaviour, delusions and mood. Although noted by the DSM-IV, these problems are not included in the definition of dementia as cognitive changes are; thus many critics believe there is too much focus on describing cognitive changes. Another problem in the DSM-IV diagnostic criteria is that a person suffering from dementia may compensate to disguise subtle changes in functioning, but unless symptoms cause considerable problems with functioning, a diagnosis of dementia is not able to be made. This can create confusion in diagnosing dementia.

While not changing significantly around diagnoses and classifications, DSM-IV-TR published in 2000 provided further information based on research and field trials. Within this publication prevalence figures of dementia were updated, codes for all the dementias except Vascular Dementia were changed, and due to considerable research in neurodegenerative processes the causes of dementia were updated. In particular the development of personality changes were highlighted in Alzheimer's disease, also data on chromosomal links and genetic markers in late onset Alzheimer's was included (American Psychiatric Association, 2009).

An older person psychiatric unit presents various mental disorders, most noticeably dementia, delirium and depression. As these diagnoses are very similar in presentation it is important for psychogeriatricians to have a classification system such as DSM-IV-TR to provide a uniform language and specific descriptions of each disorder for an accurate 
diagnosis (Gray \& Zide, 2006). Should the presentation be dementia, the clinician needs to be able to differentiate between the many types of dementia within DSM- IV-TR subtypes to obtain an accurate diagnosis.

An advantage of the DSM manual is that it constantly provides the most progressive up-to-date research on psychiatric disorders (Gray \& Zide, 2006). However, there are criticisms that there is not enough time between revisions for research to be done (Corcoran \& Walsh, 2006). For instance, there has been a considerable advance in medicine and imaging technologies (First, 2008) that, as a result, are leading to extraordinary findings within genetic and the pathogenesis of dementia that far exceeds present diagnostic tests for Alzheimer's Dementia. Unfortunately, due to the complexity of the differentiating bio-markers between the different types of dementia, there still needs to be further research before it can be included in DSM-V.

A further criticism regarding the DSM is that it allows for a person's defective behaviour to be considered a mental disorder (Gray \& Zide, 2006). Kutchins and Kirk (1996) in the title of their book concur, stating that the increasing number of diagnoses in each revision is 'making us crazy'. For example, many people forget things and have difficulty making decisions every day, but under what conditions and what reasoning is it labelled as dementia? Also, people can become disempowered by labels given to behaviour by allowing a diagnosis to be who they are rather than being someone with symptoms of a mental illness. Kutchins and Kirk (1996) agree, adding that there is a tendency with all the many health conditions now listed in DSM-IV-TR for people to be labelled, and mislabelled. On the other hand, by presenting a medical diagnosis a person may be eligible for disability or invalid benefits, a financial relief when a client has been receiving only a minimal sickness benefit (Work and Income New Zealand, 2009).

Kutchins and Kirk (1996) also note how the recognition of so many personal troubles intrigues people, thereby increasing the sales of DSM at each revision. Corcoran and Walsh (2006) also raise political issues of the DSM around the revenue made from the sale of publications, and the sales of pharmaceuticals. Pharmaceutical companies provide for a lucrative market in early medical intervention approaches to dementia (Chiu, 2005). Kaplan (2009) agrees, commenting further that of the 170 panel members that contributed to DSM-IV, 96 had financial ties to pharmaceutical companies. To remedy this there are now strict guidelines for serving on the DSM task force. No one is to hold stocks or shares in health care-related commercial ventures. Therefore no one should benefit from biased decisions.

Due to dementia being a biological disorder and not caused by psychosocial factors, Corcoran and Walsh (2006) assert that social workers have no issues concerning the diagnosis of dementia with regard to political concerns, labelling, stigma and value judgements. As previously discussed, arguably there are concerns. Although dementia is not caused by psychosocial factors (Gray \& Zide, 2006) the dementia process can be slowed or exacerbated by social and emotional factors. Often there can be conflicting opinions within families regarding the care of an elderly dementia patient. This tension may be sensed by the patient, and exacerbate symptoms. Social workers are trained to assist with family mediation, therapy and provide resources for the family. Therefore social workers offer considerable support as part of the Recovery process (Perkins, 2006). 


\section{DSM and culture}

The DSM-IV-TR format also assists with differing perspectives of mental illness within cultures, with the addition of an appendix of culturally bound syndromes, inclusion of culturally specific diagnoses, and a suggested structure for the assessment of five cultural factors (American Psychiatric Association, 2005). However, there is no description on how this information is used in assessment and diagnosis, thus context and meaning can be lost. Thakker and Ward (1998) emphasise that clinicians need to be aware that the DSM comes from a Western perspective. For example, within Maori whanau/families elders are respected and appreciated for their skills, knowledge and leadership as kaumatua (Glasgow, 2005). However, when Maori elders are diagnosed with a mental illness such as dementia within Maori culture it can be a sign of transgression, which is regarded as whakama/shameful. Consequently, the elder may not be brought in for a medical assessment in time to slow the progress of dementia (Perkins, 2006). Sensitivity to cultural issues is important along with precise diagnosis by clinicians. Key areas for social workers (Mental Health Commission, 2001; New Zealand Association of Social Workers, 2004) in Recovery are to educate and strengthen whanau, include whanau in decision making, and ensure services are offered to Maori by Maori, along with marae-based services and programmes. Browne and Broderick (1994) propose that ethnogerontological education should be added to university curricula, as knowledge of ethnicity in aging allows social workers to understand the ways in which problems can be culturally defined.

\section{DSM in relation to social work practice}

A further criticism of the DSM is that it addresses assessment and treatment first with the problem focusing on pathological; what is wrong with the client. Medical experts and interest groups, such as pharmaceutical companies, also have a tendency to locate problems within an individual, and in isolation. Green (1994) agrees stating that social matters such as ageing and mental illness are often medicalised. Working from a medical model sees dementia 'managed'. However, the elder who is in need of assistance because their family is unable to provide support and care due to family fragmentation, stress or lack of community resources (Chiu \& Chiu, 2005) is not catered for within a medical model, nor within the DSM. Frazer, Westhuis, Daley and Phillips (2009) agree and go so far as to warn social workers not to buy into the medical model that the DSM engenders as it undermines and conflicts with the paradigm of social workers, in which assessments and interventions are observed from a social level and within systems (Corcoran \& Walsh, 2006). With this in mind the American Person in Environment System Manual (Karls \& O'Keefe, 2008) (PIE) provides social workers with a holistic four-factor psychosocial classification system. Used to code, assess and provide practice, and strategy options, PIE can be used as a complement to other classification systems.

\section{Practice and interventions}

Intervention with elderly, according to Silverstone (2005), needs to go back to the traditional ecological social work perspective of the person-in-environment as opposed to present management philosophies, and 'in models of service delivery ... that are looking at the situation-in-person' (Silverstone, 2005, p.2). These philosophies seek to cut costs and are more concerned with the requirements of the agency. The concept of person-in-situation 
focuses more on the elderly person and their interactions with their environment at all levels. Kirst-Ashman and Hull (2006) maintain that empowerment and strength-based models of practice are also used with elderly. These models emphasise self-determination, strengths, resilience and combating learned helplessness (Gibson, Kunz \& Schmidt, 2005). Bertera and Bailey-Etta (2001) include the integration of spirituality and religion in elderly social work practice that supports a holistic approach. Obstacles within intervention with elderly include feelings of being intimidated by professionals, of being taken over, or of the social worker not understanding the role of informal support systems, and believing that only professionals with training can offer help. The Families Commission (2005) conclude that intervention can be complicated if a client is too embarrassed to discuss family problems, or if an elderly client has limited cognitive functioning to make decisions.

\section{Recovery perspective}

While pharmacological interventions can slow the progression of dementia and manage symptoms, a disease such as Alzheimer's is irreversible, progressive and eventually leads to death. There is no Recovery (Perkins, 2006). However, the Mental Health Commission (2001), which mandates a philosophy of Recovery within Mental Health Services (Lampshire \& Hamer, 2004), terms 'Recovery' as the ability to live well in the presence of one's mental illness. Recovery is not just about being free from symptoms but about having quality of life, as much as is possible. Dallaire, McCubbin, Carpentier \& Clement (2009) also add that Recovery is not regarded as a clinical treatment as it occurs subjectively within the individual rather than from a clinician/medical model. It is important to note that while social work focuses on autonomy and self sufficiency as part of Recovery practice (Payne, 2005) dementia progressively strips away this 'free will', the only thing we truly own as human beings according to Frankl (1985), leaving a person extremely vulnerable. Therefore, social work Recovery for elderly clients with dementia, and their families (Alzheimer's New Zealand, 2005; Mental Health Commission, 2001; Perkins, 2006) involves a holistic approach with psychosocial interventions, such as therapeutic involvement, working from the client and family's frame of reference and culture, assisting with complex social and economic problems, education around dementia, and assistance in finding meaning in the journey.

Therapeutic intervention is the main treatment for dementia, which builds on positive reinforcement of the retained strengths the client still possesses as opposed to what cannot be done anymore (Alzheimer's New Zealand, 2005). Medical reports show (Balzac, 2006) that Alzheimer's patients, despite damage to the left hemisphere of the brain, which involves language, memory and logic, can compensate by utilising the right hemisphere, which involves imagination and artistic abilities. Thus some Alzheimer's patients have been known to acquire new artistic abilities such as painting. Painting taps into the memories, thoughts and emotions from within the artist. As a result patients also present as more focused, calm and relaxed (Alzheimer's New Zealand, 2005; Sunrise Services Distributing, 2007). This is a good example of medical knowledge assisting Recovery, and of seeing the person as more than just the illness. Validation therapy is another intervention that focuses on acknowledging what the patient is feeling and their perceptions rather than trying to bring the patient back to reality through reasoning (Perkins, 2006). For example, if a patient is asking for a spouse that has died years ago, the focus would be on validating the feelings at that moment as opposed to explaining that the spouse died. This assists with Recovery principles of 'being with' a person, and validating experiences (Lampshire \& Hamer, 2004). 


\section{Recovery and caregivers}

Caregivers play a key role in the Recovery process by providing familiarity and routine for the patient, and information about the patient's history, resources and strengths. The caregivers also benefit from psychosocial input as they begin a journey from the trauma of diagnosis, to learning to live with a loved one who has dementia. Often it is a relief for family to have a diagnosis (Gray \& Zide, 2006), and education around medical aspects of dementia assists in understanding the disease and its process. Also helpful is orientation into the medical environment, the roles of various professionals encountered, as well as community resources that are available, such as Alzheimer's New Zealand (Perkins, 2006). Information can empower caregivers by allowing a measure of control over difficulties (Gray \& Zide, 2006). It is not uncommon for caregivers to feel a tremendous burden of care against the contrast of immense loss of a person they once knew. This, along with guilt, grief, fear, powerlessness, isolation as social life and friends disappear, anxious anticipation of the inevitable and despair (Goldsmith, 2008). Part of the Recovery process is to provide counselling and support on a journey towards acceptance through reframing, exploring meaning, purpose and direction (Lampshire \& Hamer, 2004). Bryden and MacKinlay (2008) concur, stating it is a time where strengths are explored and utilised, resilence is discovered, there is growth, self-realisation, and of discovering what is important in life. Caregivers are encouraged to utilise beliefs, and search within for hope (Goldsmith, 2008). The remarkable ability of the human brain from a medical perspective, and finding subjective meaning of life and death within aspects of Recovery are often given a great deal of reflection by caregivers (Bryden \& MacKinlay, 2008), and provide a stark reality against a DSM multi axis system that only provides labels and descriptions for problems.

\section{DSM-V}

DSM-V is due for publication in 2012 and its task force and work groups are already setting up symposiums for discussions on diagnoses that may be contentious (Kuhl, 2009), such as internet addiction and obesity. Nonetheless the researchers involved state that there is an attempt to have '... a more developmentally sensitive approach to diagnosis [which] should make DSM-V a more effective tool for improved patient care' (Kuhl, 2009, p.13). Due to the criticisms of DSM (Kaplan, 2009), the APA have decided to post reports and workgroup activities on the DSM website. It is claimed that in the DSM's 60-year history there has never been such transparency as the DSM-V task force invite scrutiny on goals and the criteria for change. However, Spitzer (2009) refutes this claimed transparency, stating that what little that has been published gives no information on plans for the DSM process. In addition DSM-V participants have been asked to sign confidentiality agreements to protect aspects of the process which extends beyond the publication date. This does appear to contradict the APA objective of having an effective authoritative diagnostic tool in the DSM.

Research in America shows that, apart from insurance purposes, 50 per cent of social workers use the DSM for its authoritative influence and user-friendliness. Although there has been little effort to really challenge DSM, it has been recognised as a useful balance with other classification systems such as PIE, or as an enhancement in combination with clients, strengths and resources (Frazer, Westhuis, Daley \& Phillips, 2009; Gray \& Zide, 2006). Albeit there are issues with the DSM diagnosis within dementia, Perkins (2006) does acknowledge that the DSM provides sufficient description to avoid the mild memory problems that occur within normal ageing. 
The diversification and expansion of an ageing population means gerontological social workers will need to modify and adapt practice skills and interventions at a faster rate as changes in evidence-based practices of Recovery and the DSM are disseminated more rapidly. In an era of significant research and developments in medical science that does much towards Recovery, people ultimately still require support, stories need to be told, and experiences reflected on to be able to find meaning and purpose for a life worth living. While not comparable, the DSM complements psychosocial models of Recovery. For without a diagnosis and medication there is little hope, and without hope and meaning, there is little appreciation of the moments of joy when a fleeting glimmer of recognition is seen in the eyes of a loved one.

\section{References}

Alzheimer's New Zealand. (2005). Alzheimer's disease. Retrieved March 26, 2009, from www.alzheimer's.org.nz American Psychiatric Association. (2005). Diagnostic and statistical manual of mental disorders (4th ed.). Washington DC: Author.

American Psychiatric Association. (2009). Delirium, dementia, amnestic disorder, and other cognitive disorders. Retrieved March 30, 2009, from http: / / www.psych.org/MainMenu/Research?DSMIV / DSMIVTR/DSMIVvsDSMIVTR/ SummaryofTextChangesInDSMIVTR/DeliriumDementiaAmnesticDisorderandOtherCognitiveDisorders. aspx

Bertera, E. \& Bailey-Etta, B. (2001). Physical dysfunction and social participation among racial/ethic groups of older Americans: implications for social work. In F. Ahearn. (Ed.). Issues in global aging (pp. 97-115). London: Haworth Press.

Balzac, F. (2006). Exploring the brain's role in creativity. Neuropsychiatry Reviews, 7(5).

Browne, C., \& Broderick, A. (1994). Asian and Pacific Island elders: Issues for social work practice and education. Social Work, 39(3), 252-259.

Bryden, C., \& MacKinlay, E. (2008). Dementia: A journey inwards to a spiritual self. In E. MacKinlay (Ed.)., Ageing, disability and spirituality: Addressing the challenge of disability in later life (pp. 134-144). London: Jessica Kingsley Publishers.

Chiu, E. (2005). Limitations in the current classification systems for dementia. International Psychogeriatrics 17: Supp, S17-S26.

Chiu, E., \& Chiu, H. (2005). Dementia care in Asia. International Psychogeriatrics, 1-2.

Collier, E. (2008). Historical development of psychiatric classification and mental illness. British Journal of Nursing, 17(14), 890-894.

Corcoran, J., \& Walsh, J. (2006). Clinical assessment and diagnosis in social work practice. New York: Oxford University Press.

Dallaire, B., McCubbin, M., Carpentier, N., \& Clement, M. (2009). Representations of elderly with mental health problems held by psychosocial practitioners from community and institutional settings. Social Work in Mental Health, 7(1), 139-152.

Dziegielewski, S. (2002). DSM-IV-TR in action. New York: John Wiley \& Sons.

Families Commission. (2005). Beyond zero tolerance: Key issues and future directions for family violence work in New Zealand. Retrieved May 17, 2006 from http:/ / www.familiescommission.govt.nz/download/zero-tolerance.pdf.

First, M. (2008). Changes in psychiatric diagnosis. Retrieved March 15, 2009, from http://www.psychiatrictimes. com?display / article/10168/1347847?pageNumber $=1$

Frankl, V. (1985). Man's search for meaning. New York: Washington Square Press.

Frazer, P., Westhuis, D., Daley, J., \& Phillips, I. (2009). How clinical social workers are using the DSM-IV: A national study. Social Work in Mental Health, 7(4), 325-339.

Gibson, F., Kunz, J., \& Schmidt, M. (2005). A social work perspective. In B. Haight, \& F. Gibson. Working with older adults (4th ed.) (pp. 397-415). London: Jones and Bartlett Publishers.

Glasgow, K. (2005). Ageing is living: A guide to positive ageing. Wellington: Age Concern New Zealand.

Goldsmith, M. (2008). Tracing rainbows through the rain: Addressing the challenge of dementia in later life. In E. MacKinlay (Ed.) Ageing, disability and spirituality: Addressing the challenge of disability in later life (pp. 118-133). London: Jessica Kingsley Publishers.

Gray, S., \& Zide, M. (2006). Psychopathology: A competency based treatment model for social workers. Singapore: Thomson Brooks/Cole.

Green. P. (1994). Studies in New Zealand social problems (2nd ed.) Palmerston North: The Dunmore Press.

Kaplan, A. (2009). DSM-V controversies. Retrieved March 15, 2009, from http:/ / www.psychiatrictimes.com.dsm$\mathrm{v} /$ article/10168/1364926?pageNumber $=1$

Karls, J., \& O'Keefe, M. (2008). Person-in-environment system manual (2nd ed.) Washington: NASW Press.

Kirst-Ashman, K., \& Hull, J.G. (2006). Understanding generalist practice (4th ed.) California: Thomson Learning Inc. 
Kuhl, E. (2009). Get early look at DSM-V development process. Psychiatric News, 44(4), 13.

Kutchins, H., \& Kirk, S. (1996). Making us crazy, DSM: The psychiatric bible and the creation of mental disorders. New York: The Free Press.

Lampshire, D., \& Hamer, H. (2004). Introduction to recovery. Auckland: Auckland District Health Board.

Mental Health Commission. (2001). Recovery competencies for New Zealand mental health workers. Wellington: Author.

Neurological Foundation of New Zealand. (2002). Alois Alzheimer. Retrieved March 20, 2009, from http:/ / www. neurological.org.nz/ html/ articlesDetail.php?documentCode=6942.

New Zealand Association of Social Workers. (2004). Code of ethics. Dunedin: Author.

Payne, M. (2005). Modern social work theory (3rd ed.) New York: Palgrave MacMillan.

Perkins, C. (2006). Dementia: A New Zealand guide. Auckland: Random House New Zealand.

Silverstone, B. (2005). Social work with the older people of tomorrow: Restoring the person-in-situation. Families in Society, 86(3), 309-320.

Spitzer, R. (2009). DSM-V transparency: Fact or rhetoric? Psychiatric Times, 26(3).

Statistics New Zealand. (2006). Population ageing in New Zealand. Retrieved May 18, 2006 from http: / / www.stats. govt.nz/ products-and-services / Articles / pop-ageing-Feb00.htm.

Sunrise Services Distributing. (2007). Alzheimer's and dementia sufferers respond to water color painting: Improving quality of life for alzheimer's and dementia sufferers. Retrieved March 28, 2009, from http: / / www.sunrisedistrib. com/ArtforAlzheimer's.html.

Thakker, J., \& Ward, T. (1998). Culture and classification: The cross-cultural application of the DSM-IV. Clinical Psychology Review, 18(5), 501-529.

Thompson, N. (2001). Anti-discriminatory practice (3rd ed.) New York: Palgrave.

Work and Income New Zealand. (2009). A-Z Benefits. Retrieved April 13, 2009, from http: / / workandincome.govt. $\mathrm{nz} /$ individuals/a-zbenefits/index.html. 